\title{
RESEARCH
}

Open Access

\section{PINK1 contained in huMSC-derived exosomes prevents cardiomyocyte mitochondrial calcium overload in sepsis via recovery of mitochondrial $\mathrm{Ca}^{2+}$ efflux}

Qin Zhou ${ }^{1 \dagger}$, Min Xie ${ }^{1,2+}$, Jing Zhu ${ }^{1,2}$, Qin Yi ${ }^{1,2}$, Bin Tan ${ }^{1,2}$, Yasha Li $i^{1,2}$, Liang Ye $e^{1,2}$, Xinyuan Zhang ${ }^{1,2}$, Ying Zhang ${ }^{1,2}$, Jie $\operatorname{Tian}^{1,3}$ and HaO $\mathrm{Xu}^{2,4^{*}}$ (iD

\begin{abstract}
Background: Sepsis is a systemic inflammatory response to a local severe infection that may lead to multiple organ failure and death. Previous studies have shown that $40-50 \%$ of patients with sepsis have diverse myocardial injuries and 70 to $90 \%$ mortality rates compared to $20 \%$ mortality in patients with sepsis without myocardial injury.

Therefore, uncovering the mechanism of sepsis-induced myocardial injury and finding a target-based treatment are immensely important.

Objective: The present study elucidated the mechanism of sepsis-induced myocardial injury and examined the value of human umbilical cord mesenchymal stem cells (huMSCs) for protecting cardiac function in sepsis.

Methods: We used cecal ligation and puncture (CLP) to induce sepsis in mice and detect myocardial injury and cardiac function using serological markers and echocardiography. Cardiomyocyte apoptosis and heart tissue ultrastructure were detected using TdT-mediated dUTP Nick-End Labeling (TUNEL) and transmission electron microscopy (TEM), respectively. Fura-2 AM was used to monitor $\mathrm{Ca}^{2+}$ uptake and efflux in mitochondria. FQ-PCR and Western blotting detected expression of mitochondrial $\mathrm{Ca}^{2+}$ distribution regulators and PTEN-induced putative kinase 1 (PINK1). JC-1 was used to detect the mitochondrial membrane potential $(\Delta \psi \mathrm{m})$ of cardiomyocytes.

\footnotetext{
* Correspondence: Ib7pl@163.com

${ }^{\dagger}$ Qin Zhou and Min Xie contributed equally to this work.

${ }^{2}$ Chongqing Key Laboratory of Pediatrics, Chongqing, People's Republic of China

${ }^{4}$ Department of Clinical Laboratory, Ministry of Education Key Laboratory of Child Development and Disorders, National Clinical Research Center for Child Health and Disorders (Chongqing), China International Science and

Technology Cooperation base of Child development and Critical Disorders, Children's Hospital of Chongqing Medical University, Box 136, No. 3

Zhongshan RD, Yuzhong district, Chongqing 400014, People's Republic of China

Full list of author information is available at the end of the article
}

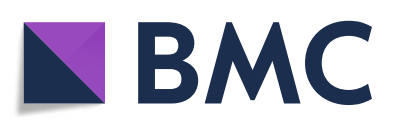

(- The Author(s). 2021 Open Access This article is licensed under a Creative Commons Attribution 4.0 International License, which permits use, sharing, adaptation, distribution and reproduction in any medium or format, as long as you give appropriate credit to the original author(s) and the source, provide a link to the Creative Commons licence, and indicate if changes were made. The images or other third party material in this article are included in the article's Creative Commons licence, unless indicated otherwise in a credit line to the material. If material is not included in the article's Creative Commons licence and your intended use is not permitted by statutory regulation or exceeds the permitted use, you will need to obtain permission directly from the copyright holder. To view a copy of this licence, visit http://creativecommons.org/licenses/by/4.0/. The Creative Commons Public Domain Dedication waiver (http://creativecommons.org/publicdomain/zero/1.0/) applies to the data made available in this article, unless otherwise stated in a credit line to the data. 
(Continued from previous page)

Results: We found that expression of PINK1 decreased in mouse hearts during sepsis, which caused cardiomyocyte mitochondrial $\mathrm{Ca}^{2+}$ efflux disorder, mitochondrial calcium overload, and cardiomyocyte injury. In contrast, we found that exosomes isolated from huMSCs (huMSC-exo) carried Pink1 mRNA, which could be transferred to recipient cardiomyocytes to increase PINK1 expression. The reduction in cardiomyocyte mitochondrial calcium efflux was reversed, and cardiomyocytes recovered from injury. We confirmed the effect of the PINK1-PKA-NCLX axis on mitochondrial calcium homeostasis in cardiomyocytes during sepsis.

Conclusion: The PINK1-PKA-NCLX axis plays an important role in mitochondrial calcium efflux in cardiomyocytes. Therefore, PINK1 may be a therapeutic target to protect cardiomyocyte mitochondria, and the application of huMSC-exo is a promising strategy against sepsis-induced heart dysfunction.

Keywords: Sepsis, Cardiac dysfunction, PINK1, Calcium overload, Mitochondrial Ca ${ }^{2+}$ efflux

\section{Introduction}

Sepsis is a systemic inflammatory response to a local severe infection that may lead to multiple organ failure and death, especially in patients with cardiac dysfunction, which increases mortality to $70-90 \%$ compared to patients without cardiac dysfunction [1]. Sepsis-induced cardiac injury or dysfunction subsequently contributes to cardiovascular collapse, which results in poor perfusion of blood into multiple tissues [2]. Therefore, examining the mechanism of sepsis-induced cardiac injury and finding a way to protect the heart from injury during sepsis would provide beneficial effects on mortality in this complex disease.

The mechanisms underlying cardiac dysfunction in sepsis are not completely understood but include the following main mechanisms: (a) increased release of proinflammatory cytokines [3], (b) apoptotic myocardial cell death via activation of extrinsic and intrinsic pathways, (c) metabolic disorders and inducible nitric oxide synthase (NOS)-dependent action [4], (d) cardiacdepressant action of lysozyme c primarily originating from disintegrating neutrophils and monocytes [5], and (e) mitochondrial dysfunction [6, 7]. Previous studies observed mitochondrial structural abnormalities at the early stage of sepsis, and mitochondrial respiration function was reduced significantly and caused a reduction in ATP production, which led to myocardial contractility dysfunction $[8,9]$. However, there are few studies on the mechanism of cardiomyocyte mitochondrial dysfunction during sepsis.

In the early stage of sepsis, an abundance of $\mathrm{Ca}^{2+}$ flow into the cytoplasm of cardiomyocytes from the extracellular space, and the mitochondrial uptake creates an overload of $\mathrm{Ca}^{2+}$ in the matrix, which ultimately induces mitochondrial calcium overload. Mitochondrial injury induced by calcium overload is an important mechanism of heart dysfunction in sepsis. The obstruction of mitochondrial $\mathrm{Ca}^{2+}\left({ }_{\mathrm{m}} \mathrm{Ca}^{2+}\right)$ efflux mediated by the mitochondrial $\mathrm{Na}^{+} / \mathrm{Ca}^{2+}$ exchanger (NCLX) is an important factor in mitochondrial calcium overload [10]. The activity of NCLX is regulated by several proteins and kinases, such as stomatin-like protein 2 (SLP-2) and protein kinase $\mathrm{C}(\mathrm{PKC})[11,12]$. In a cell model of Parkinson's disease related to mutations in PTEN-induced putative kinase 1 (PINK1), the failure of mitochondrial $\mathrm{Ca}^{2+}$ efflux in neurons deficient in PINK1 was linked to the impaired activity of NCLX, which resulted in mitochondrial $\mathrm{Ca}^{2+}$ overload and suggested that PINK1 regulated the activity of NCLX [13, 14]. Therefore, whether disorders of PINK1 and NCLX underlie cardiomyocyte mitochondrial dysfunction and whether reducing mitochondrial $\mathrm{Ca}^{2+}$ overload would rescue the cardiac injury are worthy of investigation.

Mesenchymal stem cells (MSCs) effectively reduce mortality and improve myocardial function in animal models of sepsis [15-17]. However, MSCs primarily migrate to the lung and liver after systemic infusion in these models, and few cells are detected in cardiac tissue $[18,19]$. Therefore, MSC-induced cardiac benefits during sepsis may not be related to local actions but to paracrine effects from a distance [20]. The beneficial effects of MSCs are mediated by paracrine factors, such as cytokines, growth factors, and extracellular vesicles. Exosomes are extracellular vesicles that mediate local and systemic cell-to-cell communication. These vesicles are 30-100-nm-sized membrane vesicles that transfer specific sets of functional RNAs (miRNAs, mRNAs, and lncRNAs) and proteins to recipient cells [21]. Several studies have shown that MSC-derived exosomes (MSCexos) improved recovery in animal models of liver fibrosis, kidney failure, and myocardial ischemia/reperfusion injury [20, 22, 23]. Xiaohong Wang et al. reported that MSC-exos also contributed to cardioprotective effects in sepsis [20], but how MSC-exos exert cardioprotective effects during sepsis was not elucidated.

Based on the current theoretical framework, we hypothesized that sepsis-induced cardiac dysfunction may be caused by cardiomyocyte mitochondrial calcium 
overload, and MSC-exos would rescue injured mitochondria by reducing calcium overload. The present study examined the mechanism of cardiomyocyte mitochondrial calcium overload during sepsis and investigated whether MSC-exos protected heart function during sepsis by reducing mitochondrial damage and whether MSC-exos repaired mitochondrial damage via PINK1 and NCLX.

\section{Methods}

\section{Animals and sepsis model}

C57BL/6 mice aged 6-8 weeks were obtained from and raised at Chongqing Medical University. Mice were randomly assigned to three groups: sham, cecal ligation and puncture (CLP) and CLP + exo. Sixteen mice were used for each group: 6 mice were used to detect serum biomarkers and ACM isolation, 3 of these mice underwent echocardiography before serum collection, and 10 mice were used to measure survival rate. CLP was performed to establish a model of sepsis [24]. Briefly, the animals were anesthetized with pentobarbital $(1 \%, 50 \mathrm{mg} / \mathrm{kg}$ body weight i.p.), and small scissors were used to make the incision and gain entry into the peritoneal cavity. The cecum was located, exteriorized, ligated, and punctured using a 26-gauge needle at the designated position to induce mid-grade sepsis. The cecum was relocated into the abdominal cavity, and the incision was closed. The sham group was exposed to the same surgery, but the cecum was not ligated or punctured. The CLP + exo group were treated with exosomes isolated from human MSCs (huMSCs) after CLP. Serum biomarkers and echocardiography were used to evaluate sepsisinduced heart injury [9].

\section{Echocardiography}

Mice in each group were administered an anesthetic at the corresponding time point after modeling. The chest skin was depilated, and the mice were placed on a plate to maintain anesthesia and sedation. Transthoracic echocardiography was performed using a highfrequency, high-resolution digital imaging system (Vevo 2100 Imaging System, Fujifilm Visualsonics, Canada) with a transducer probe (VisualSonics 550D, Fujifilm Visualsonics, Canada). A thick gel was applied to the chest, the probe was placed on the left edge of the sternum, and two-dimensional B-mode ultrasound was used to display the standard left ventricular short-axis view. The probe was moved to the level of the papillary muscles, and M-mode ultrasound was used to record the left ventricular motion curve. A single trained individual performed all echocardiograms.

\section{Serological markers of myocardial injury detection}

At least $500 \mu \mathrm{L}$ of whole blood per mouse was collected in heparin, and the plasma was collected after centrifugation at $8000 \mathrm{rpm}$ for $10 \mathrm{~min}$. The levels of $\alpha$ hydroxybutyrate dehydrogenase $(\mathrm{HBDH})$ and creatine kinase (CK) in plasma were measured using an automatic biochemical analyzer (C701, Roche, Switzerland). Cardiac troponin-I (cTnI) was quantified using ELISA (E-EL-M1203c, Elabscience, China).

\section{Cell culture and treatments}

The huMSCs used in our study were obtained from Chongqing Stem Cell Therapy Engineering and Technology Center and cultured in DMEM/F12 supplemented with $10 \%$ fetal bovine serum (FBS; Millipore, USA). Adult mouse cardiomyocytes (ACMs) were isolated from adult mouse hearts and cultured in dishes or plates precoated with mouse laminin [25]. Human ventricular myocyte AC16 cells (Otwo Biotech, ShenZhen, China) were cultured in DMEM supplemented with $10 \%$ fetal bovine serum and treated with lipopolysaccharide (LPS, $5 \mu \mathrm{g} / \mathrm{ml}$, Solarbio, L8880, China) to create an in vitro sepsis model. The AC16 cells were treated with forskolin $(20 \mu \mathrm{M}, 12 \mathrm{~h}$, Selleck, S2449, USA) or the protein kinase A (PKA) inhibitor H89 $(5 \mu \mathrm{M}, 12 \mathrm{~h}$, Selleck, S1582, USA).

\section{Transient transfection with siRNA}

The huMSCs were transfected with siRNA according to the manufacturer's instructions. Transfection of siRNA into huMSCs was performed using RNAiMax reagent (Thermo Fisher Scientific, USA) at a final concentration of $100 \mathrm{nM}$. Cellular RNA, protein, and culture medium supernatant collection were performed $48 \mathrm{~h}$ after transfection. The siRNA sequence for Pink1 was 5'GGCTGGTGATCGCAGATTT-3'.

\section{Isolation and characterization of exosomes}

The huMSCs were maintained under normal conditions until they reached 70 to $80 \%$ confluency. Twenty-four hours before the collection of exosomes from huMSCs and huMSCs transfected with Pink1 siRNA, the medium was replaced with medium containing $10 \%$ exosomedepleted FBS. Supernatants were collected and centrifuged successively at $300 \times g$ for $10 \mathrm{~min}, 2000 \times g$ for 10 $\mathrm{min}$, and $10,000 \times \mathrm{g}$ for $30 \mathrm{~min}$, and the supernatants were transferred to a new tube at each step. The final supernatant was ultracentrifuged at $100,000 \times g$ for $70 \mathrm{~min}$, and the pellet was washed in PBS and ultracentrifuged at $100,000 \times g$ for another $70 \mathrm{~min}$ [26]. The pellet was resuspended in PBS and quantified using the BCA assay. The mice were treated with $2 \mu \mathrm{g} / \mathrm{g}$ exosomes at $0 \mathrm{~h}$ and $6 \mathrm{~h}$ after CLP respectively and cocultured with cells at $2 \mu \mathrm{g} /$ $\mathrm{ml}$. The quality of the exosomes was confirmed using 
transmission electron microscopy (TEM), particle size assessment (ZEN3600, Malvern, UK), and exosome markers. To monitor exosome trafficking, exosomes were labeled with $\mathrm{PKH} 26$ fluorescent dye using the PKH26 fluorescent cell linker kit (Sigma-Aldrich, MKCJ1898).

\section{Fluorescence quantitative PCR}

Total RNA was isolated from rat hearts using TRIzol (Takara, Japan) according to the manufacturer's instructions. cDNA was generated using $1 \mu \mathrm{g}$ RNA with a PrimeScriptTM RT Reagent Kit (Takara, Japan). FQ-PCR was performed using TB Green (Takara, Japan) with a CFX-96 (BIO-RAD, USA). We evaluated samples for mRNA expression of Mcu, Micul, Nclx, and Pink1. All experimental samples were analyzed in triplicate and averaged. To calculate the fold change in mRNA expression, the $2^{-\Delta \Delta \mathrm{Ct}}$ method was used.

\section{Western blotting}

Proteins were isolated from rat hearts using lysis buffer (KeyGEN BioTECH, China) with a protease and phosphatase inhibitor cocktail solution (KeyGEN BioTECH, China). Thirty micrograms of protein was loaded on a 10\% PAGE Gel Fast Preparation Kit (PG112, Epizyme Biotech, China). Proteins were separated and transferred to PVDF membranes (Millipore, USA). Membranes were incubated in TBST skim milk blocking buffer for $1 \mathrm{~h}$ at room temperature (RT) followed by primary antibodies overnight at $4{ }^{\circ} \mathrm{C}$. The following primary antibodies were used: CD81 (sc-166,029, Santa Cruz, USA), Alix (sc-53, 540, Santa Cruz, USA), MCU (26312-1-AP, Proteintech, USA), MICU1 (ab224161, Abcam, England), NCLX (ab83551, Abcam, England), PINK1 (23274-1-AP, Proteintech, USA), and GAPDH (AF7021, Affinity Biosciences, USA). Membranes were washed with TBST three times, incubated with secondary antibodies (701051, Zen Bioscience, China; ZB-2301, ZSGB-BIO, China) for $1 \mathrm{~h}$ at RT and washed with TBST three times. Membranes were probed using a chemiluminescence kit (Millipore, USA) and an Image system (Bio-Rad, USA).

\section{Protein kinase $A$ (PKA) activity assay}

PKA activity was examined using PKA Kinase Assay Kits, Type I (Immunechem, ICP0216) according to the manufacturer's protocol. Briefly, the supernatant $(50 \mu \mathrm{l} /$ well) of samples was added to the substrate plate containing kinase assay dilution buffer. ATP $(10 \mu \mathrm{l} /$ well $)$ was added to initiate the kinase reaction at $30{ }^{\circ} \mathrm{C}$ for $90 \mathrm{~min}$. The reaction solution was removed and anti-p-substrate antibodies $(40 \mu \mathrm{l} /$ well $)$ were incubated for $60 \mathrm{~min}$ at RT. Goat anti-rabbit IgG HRP was used as a secondary antibody, and TMB solution was used to indicate reaction activity. Relative kinase activity was detected at OD450 and normalized to the protein concentration, which was measured using the BCA method.

\section{TdT-mediated dUTP Nick-End Labeling (TUNEL)}

The tissue was fixed in $4 \%$ paraformaldehyde for at least $24 \mathrm{~h}$, embedded in paraffin and sectioned into $4-\mu \mathrm{m}$ slices. Apoptosis was assessed using a TUNEL kit (KGA702-1, KeyGEN BioTECH, China) according to the manufacturer's instructions. Images were acquired on a Nikon microscope (90i, Japan).

\section{TEM}

Heart tissue was fixed with a $4 \%$ glutaraldehyde solution for $2 \mathrm{~h}$ and postfixed with $1 \%$ osmium tetroxide for $2 \mathrm{~h}$ at $4{ }^{\circ} \mathrm{C}$. The fixed tissue was rinsed with distilled water, dehydrated in an ethanol and methanol gradient, and embedded in epoxy resin. The samples were sectioned and contrast-stained for imaging. TEM images were acquired at random locations throughout the samples. Micrographs were taken with a transmission electron microscope (H-7500).

\section{Evaluation of ${ }_{\mathrm{m}} \mathrm{Ca}^{2+}$ uptake and efflux}

ACMs or AC16 (300000) cells were transferred to an intracellular-like medium containing $120 \mathrm{mM} \mathrm{KCl,} 10$ $\mathrm{mM} \mathrm{NaCl}, 1 \mathrm{mM} \mathrm{KH_{2 }} \mathrm{PO}_{4}, 20 \mathrm{mM}$ HEPES-Tris, $3 \mathrm{mM}$ thapsigargin, $80 \mu \mathrm{g} \mathrm{ml}^{-1}$ digitonin, protease inhibitors, and $10 \mu \mathrm{M}$ succinate at $\mathrm{pH} 7.2$. Fura- $2 \mathrm{AM}(1 \mu \mathrm{M})$ was added to monitor extramitochondrial $\mathrm{Ca}^{2+}$. Fluorescence signals were monitored at excitations of 340 and $380 \mathrm{~nm}$ and an emission of $510 \mathrm{~nm}$ for Fura-2 to calculate ratiometric changes. At $60 \mathrm{~s}$, a $20-\mu \mathrm{M} \mathrm{Ca}{ }^{2+}$ bolus was added. Clearance of extramitochondrial $\mathrm{Ca}^{2+}$ was representative of $\mathrm{mCa}^{2+}$ uptake. At $260 \mathrm{~s}, 1 \mu \mathrm{M}$ Ru360 (MCU inhibitor) was added to inhibit uptake and allow for quantification of $\mathrm{Ca}^{2+}$ efflux. At $360 \mathrm{~s}, 10 \mu \mathrm{M}$ CGP-37157 (NCLX inhibitor) was added to block $\mathrm{mCa}^{2+}$ efflux. FCCP was added at the completion of the experiment. All experiments were performed at $37^{\circ} \mathrm{C}$ and recorded on a Cytation 5 (Biotek, USA). The details were reported previously [10].

\section{Mitochondrial membrane potential assay}

The mitochondrial membrane potential was measured using a mitochondrial membrane potential assay kit (JC1, Beyotime Biotech, China) according to the manufacturer's instructions. Cells were incubated with medium mixed with JC-1 working fluid in a $37^{\circ} \mathrm{C}$ incubator for $20 \mathrm{~min}$. The cells were washed with JC-1 buffer three times and replaced with fresh medium. Fluorescence was captured using confocal microscopy (A1R, Nikon, Japan). The ratio of red and green fluorescence was analyzed by an NIS-Elements system (Nikon, Japan). A high 
ratio indicated a high mitochondrial membrane potential.

\section{Statistical analysis}

Each experiment was repeated at least three times. All data are expressed as the means $\pm \mathrm{SD}$, and the statistical evaluations were performed using $t$ tests with independent samples. One-way analysis of variance (ANOVA) with Tukey's multiple comparisons test was used between groups. SPSS 17.0 software (SPSS Inc., Armonk, NY, USA) was used for statistical analyses. For all analyses, a value of $p<0.05$ was considered significant.

\section{Results}

\section{Sepsis-induced heart injury and huMSCs-exo showed} cardioprotective effects

HuMSCs-exo were isolated and purified via differential ultracentrifugation and assessed using TEM, exosome markers, and particle size (Fig. 1a-c, FigS1 A). We used CLP to investigate the cardioprotective effects of huMSCs-exo in septic mice. A 2- $\mu \mathrm{g} / \mathrm{g}$ concentration of huMSCs-exo was intraperitoneally injected $0 \mathrm{~h}$ and $6 \mathrm{~h}$ after CLP, followed by testing at $12 \mathrm{~h}$ after CLP. We tracked the labeled exosomes by Confocal Microscopy (FigS2) and confirmed the cardiomyocyte uptake the exosomes at $12 \mathrm{~h}$ after CLP.

We found that the serum markers of cardiomyocyte injury (HBDH, CK and cTnI) increased significantly after CLP (Fig. 1d, HBDH: CLP $12 \mathrm{~h}$ group $1210 \pm 246.2$ versus sham group $158.2 \pm 14.41, p<0.0001$; CK: CLP $12 \mathrm{~h}$ group $14,238 \pm 2293$ versus sham group $2229 \pm 582.7$, $p<0.0001$; cTnI: CLP $12 \mathrm{~h}$ group $115.1 \pm 30.8$ versus sham group $36.35 \pm 7.167, p<0.0001$ ), which indicated that sepsis induced cardiomyocyte injury in the first 12 h. In contrast, these markers decreased markedly in the huMSCs-exo treatment group (Fig. 1d, HBDH: CLP 12 $\mathrm{h}+$ exo group $647 \pm 162.2$ versus CLP $12 \mathrm{~h}$ group $1210 \pm$ 246.2, $p=0.0001$; CK: CLP $12 \mathrm{~h}+$ exo group $6884 \pm 1051$ versus CLP 12 h group $14,238 \pm 2293, p<0.0001$; cTnI: CLP $12 \mathrm{~h}+$ exo group $70.66 \pm 18.4$ versus CLP $12 \mathrm{~h}$ group $115.1 \pm 30.8, p=0.0063$ ), which indicated that treatment with huMSCs-exo effectively mitigated sepsisinduced cardiomyocyte injury. We detect heart function measured by echocardiography during sepsis (Fig. 1e, f, Table S1). The results showed that EF decreased notably at $12 \mathrm{~h}$ after CLP, and huMSCs-exo significantly improved EF compared to the CLP group (Fig. 1e, f, CLP $12 \mathrm{~h}$ group $72.11 \pm 4.231 \%$ versus sham group $91.49 \pm$ $2.925 \%, p=0.0012$, and CLP $12 \mathrm{~h}+$ exo group $87.84 \pm$ $3.127 \%, p=0.0035)$. These results indicated that huMSCs-exo prevented sepsis-induced heart function disorder. CLP mouse survival also increased significantly after huMSC-exo treatment compared to the untreated group (Fig. 1g).

\section{Cardiomyocyte mitochondrial damage caused by sepsis can be reversed by huMSCs-exo}

To investigate the extent of cardiomyocyte injury in early sepsis, we used TUNEL to measure cardiomyocyte apoptosis. We found that serum markers of cardiomyocyte injury increased significantly, but there was almost no apoptosis in cardiomyocytes $12 \mathrm{~h}$ after CLP, and some cardiomyocytes did not undergo apoptosis until $24 \mathrm{~h}$ after CLP (Fig. 2a). This result suggests that cardiomyocytes were in the early stage of apoptosis $12 \mathrm{~h}$ after sepsis, and some factors increased cardiomyocyte membrane permeability and resulted in cardiac-specific protein release.

To reveal the reason for increased injury markers and identify changes in cardiomyocytes during the first $12 \mathrm{~h}$ of sepsis, we observed cardiomyocyte ultrastructure using TEM. We observed a disorderly myocardial myofibril arrangement, and the band area was blurred, broken, or dissolved $12 \mathrm{~h}$ after CLP despite the absence of cardiomyocyte apoptosis. We also observed that cardiomyocyte mitochondrial swelling, cristae disorganization, and cristae number decreased, which indicated that cardiomyocyte mitochondria were seriously injured during the first $12 \mathrm{~h}$ of sepsis (Fig. 2b). One of the main characteristics of mitochondrial dysfunction is reduced ATP production, which was consistent with our results, and we found that ATP production was significantly reduced $12 \mathrm{~h}$ after CLP (Fig. 2c, CLP $12 \mathrm{~h}$ group $0.6058 \pm 0.07267$ versus sham group $1(100 \%), p=0.0005)$.

We hypothesized that the cardioprotective effects of huMSC-exo were mediated via mitochondria protection. Therefore, we examined cardiomyocyte ultrastructure using TEM after treatment with huMSC-exo and found that myocardial myofibrils were arranged in order with no broken or dissolved myofibrils. The mitochondria morphology was similar to the sham group, with only a few mitochondria showing a decreased number of cristae (Fig. 2b). This observation indicates that huMSC-exo effectively prevented cardiomyocyte mitochondrial injury in the first $12 \mathrm{~h}$ of sepsis. The ATP production of cardiomyocytes in the treatment group $12 \mathrm{~h}$ after CLP also increased to a similar level as the sham group (Fig. 2c, CLP $12 \mathrm{~h}+$ exo group $1.01 \pm 0.1918$ versus CLP $12 \mathrm{~h}$ group $0.6058 \pm 0.07267, p=0.0004$ ), which may be a benefit from the undamaged mitochondria protected by huMSC-exos.

\section{Cardiomyocyte mitochondrial $\mathrm{Ca}^{2+}\left({ }_{\mathrm{m}} \mathrm{Ca}^{2+}\right)$ efflux was obstructed during sepsis and reversed by huMSCs-exo} To investigate how mitochondrial calcium overload of cardiomyocytes occurred in sepsis, we assessed the capacity of $\mathrm{Ca}^{2+}$ uptake and efflux in cardiomyocyte mitochondria. Adult cardiomyocytes (ACMs) isolated from 


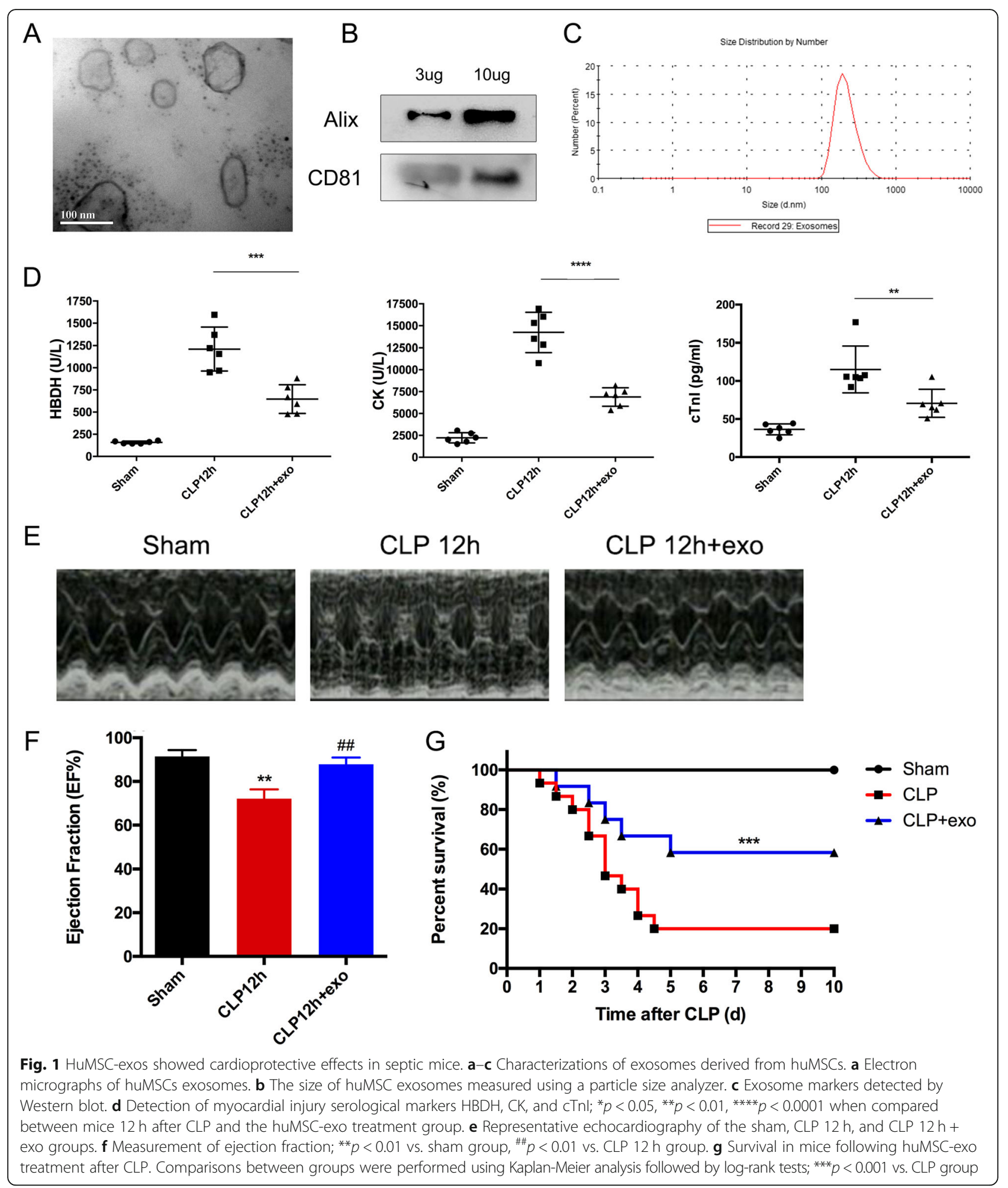

sham-, CLP-, and huMSC-exo-treated mice were transferred to an intracellular-like medium, and $1 \mu \mathrm{M}$ Fura2AM was added to monitor extramitochondrial $\mathrm{Ca}^{2+}$. Thapsigargin was added to prevent SR and ER $\mathrm{Ca}^{2+}$ uptake. Therefore, the changes in the fluorescence intensity indicated $\mathrm{Ca}^{2+}$ influx or efflux into/out of the mitochondria. The results showed that after the $\mathrm{Ca}^{2+}$ bolus was added, the time for extramitochondrial $\mathrm{Ca}^{2+}$ to reach the low concentration occurred earlier in the CLP group than the sham group (Fig. 3a, b $60 \mathrm{~s}-260 \mathrm{~s}$ ), which 

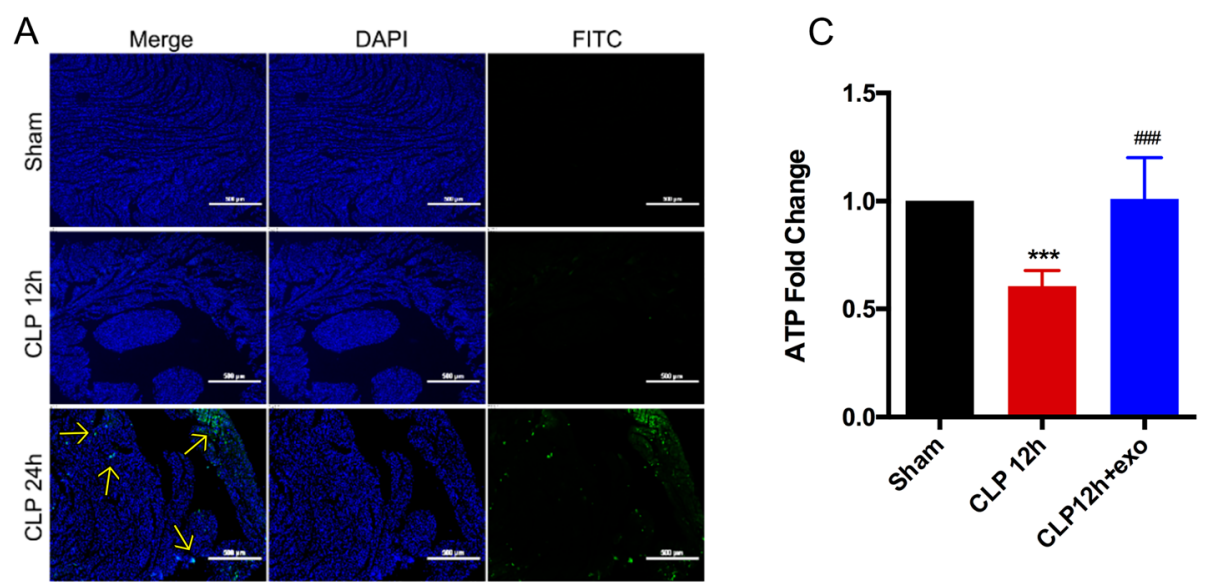

B

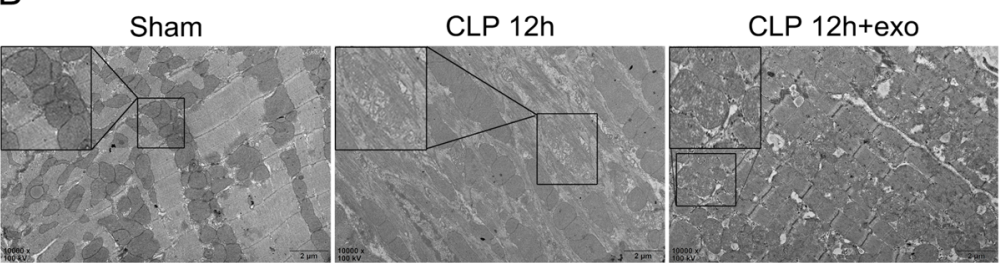

Fig. 2 Sepsis-induced heart dysfunction is caused by mitochondrial injury, which is reversed by huMSCs-exo. a Representative TUNEL staining for the detection of apoptotic cardiomyocytes (green dots indicated by arrows); nuclei were stained with DAPI (blue). $\mathbf{b}$ After CLP for $12 \mathrm{~h}$ and treatment with huMSCs-exo, the mitochondrial architecture was observed under transmission electron microscopy (TEM) and imaged at a $\times$ 10,000 magnification (scale bars, $2 \mu \mathrm{m}$ ); the box in the image is a local magnification. c The cardiomyocyte ATP content in mice $12 \mathrm{~h}$ after the CLP and treatment with huMSC-exo was measured using a bioluminescent assay system; ${ }^{* *} p<0.001$ vs. sham group, \#\#\#p $<0.001$ vs. CLP $12 \mathrm{~h}$ group

indicated that mitochondrial $\mathrm{Ca}^{2+}$ uptake in the CLP group was slightly faster than in the sham group. After MCU was inhibited with Ru360 at $260 \mathrm{~s}-360 \mathrm{~s}, \mathrm{Ca}^{2+}$ uptake in the mitochondria was blocked, and the increase in extramitochondrial $\mathrm{Ca}^{2+}$ was representative of $\mathrm{mCa}^{2+}$ efflux. These results showed that the peak concentration of extramitochondrial $\mathrm{Ca}^{2+}$ was much lower in the CLP group than the sham group during the same period of time, which indicates that the $\mathrm{mCa}^{2+}$ efflux rate decreased significantly in the CLP group (Fig. 3a, b $260 \mathrm{~s}-$ $360 \mathrm{~s}, \mathrm{~d}$, CLP $12 \mathrm{~h}$ group $3.437 \pm 0.4563$ versus sham group $7.047 \pm 1.274, p=0.0083)$. In contrast, we found that although $\mathrm{Ca}^{2+}$ uptake by mitochondria in the huMSC-exo treatment group was not different from the CLP group (Fig. 3c $60 \mathrm{~s}-260 \mathrm{~s}$ ), the ${ }_{\mathrm{m}} \mathrm{Ca}^{2+}$ efflux rate increased to the level of the sham group (Fig. 3c $260 \mathrm{~s}-$ $360 \mathrm{~s}, \mathrm{~d}, \mathrm{CLP} 12 \mathrm{~h}+$ exo group $6.216 \pm 0.9313$ versus CLP $12 \mathrm{~h}$ group $3.437 \pm 0.4563, p=0.0268$ ), which suggests that huMSC-exo maintained normal $\mathrm{mCa}^{2+}$ efflux of cardiomyocyte mitochondria during sepsis.

Mitochondria regulate $\mathrm{maa}^{2+}$ distribution in two ways: cytoplasmic $\mathrm{Ca}^{2+}$ uptake is driven by $\Delta \Psi \mathrm{m}$ and mediated primarily by $\mathrm{MCU}$ and MICU1 [27], and $\mathrm{m}^{2{ }^{2+}}$ efflux mediated by mitochondrial NCLX [28]. We assessed the mRNA expression of Mcu, Micul, and Nclx $12 \mathrm{~h}$ after
CLP and treatment with huMSCs-exo; the results showed there was little difference between these groups (Fig. 3e Mcu: sham group 1 (100\%) versus CLP $12 \mathrm{~h}$ group $1.102 \pm 0.2, p=0.428$ and CLP12h + exo group $1.157 \pm 0.29 p=0.686$; Micu1: sham group 1 (100\%) versus CLP $12 \mathrm{~h}$ group $1.01 \pm 0.162, \quad p=0.917$ and CLP12h + exo group $1.142 \pm 0.28, p=0.648$; Nclx: sham group 1 (100\%) versus CLP $12 \mathrm{~h}$ group $1.016 \pm 0.064$, $p=0.693$ and CLP12h + exo group $1.172 \pm 0.294, p=$ 0.545). The protein level of MCU, MICU1, and NCLX has also no difference between these groups (Fig. 3f, FigS1. B). These results indicate that the reason for abnormal ${ }_{\mathrm{m}} \mathrm{Ca}^{2+}$ efflux $12 \mathrm{~h}$ after CLP may be suppression of NCLX activity.

\section{HuMSC-exo increased expression of PINK1 in cardiomyocytes during sepsis}

Previous reports indicated that PINK1 regulated the activity of NCLX in neurons, but whether PINK1 also regulated the activity of NCLX in cardiomyocytes was not known. We found that expression of PINK1 decreased significantly $12 \mathrm{~h}$ after CLP (Fig. 4a), which is consistent with the abnormal $\mathrm{mCa}^{2+}$ efflux results and suggests that the decrease in PINK1 expression in cardiomyocytes is associated with a reduction in NCLX activity. We also 


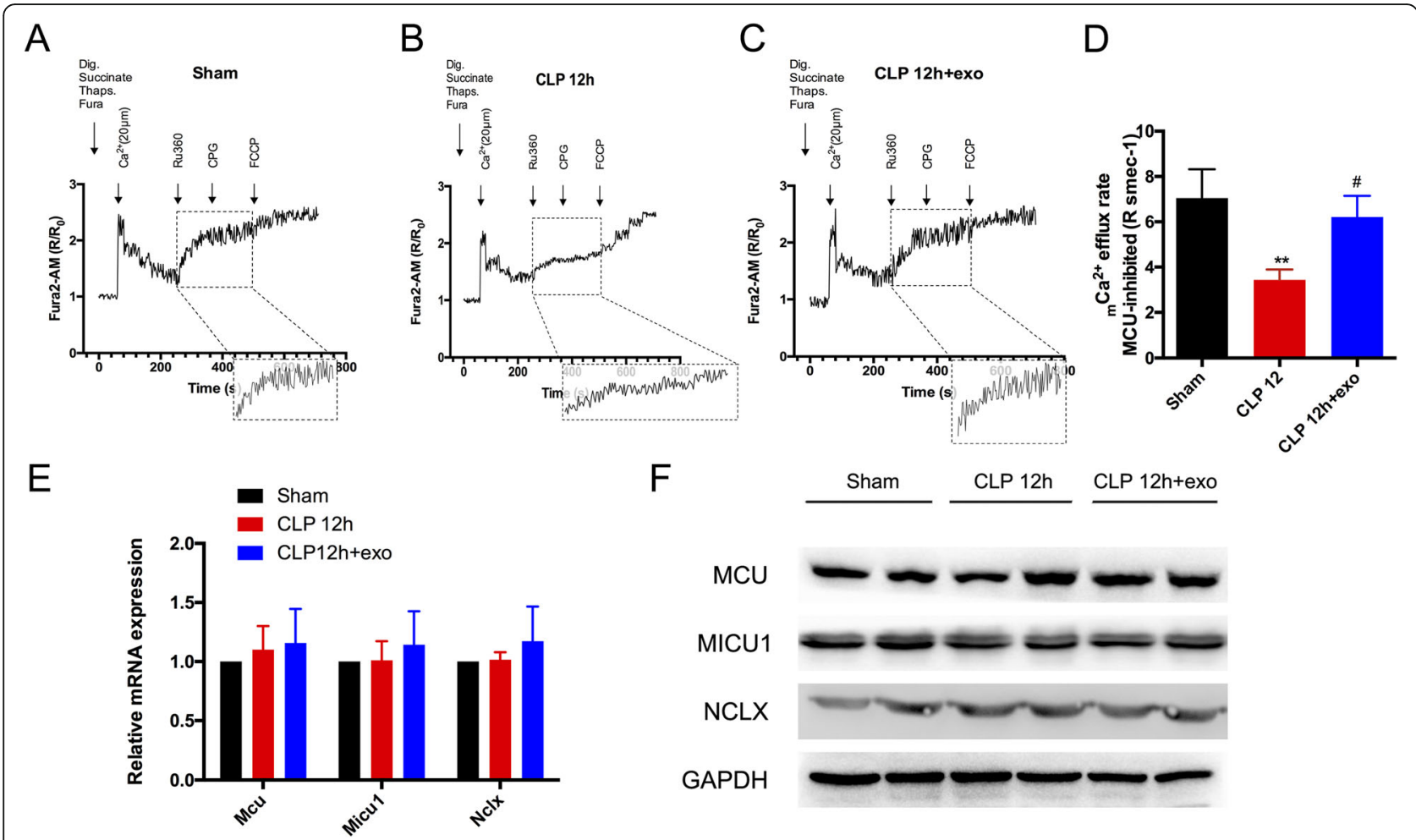

Fig. 3 Assessment of cardiomyocyte mitochondrial $\mathrm{Ca}^{2+}\left(\mathrm{mCa}^{2+}\right)$ uptake, efflux, and the corresponding regulator. a- $\mathbf{c} \mathrm{mCa}^{2+}$ uptake and efflux in isolated permeabilized ACMs; dig.: digitonin, thaps.: thapsigargin, Ru360: MCU inhibitor, CGP: NCLX inhibitor; the box shows a magnified ${ }_{m} \mathrm{Ca}^{2+}$ efflux tracing (260 s-360 s); R indicates the ratio of the ratiometric reporter Fura-2 (340/380 nm excitation and $510 \mathrm{~nm}$ emission). R/Ro indicates the ratio at each time point over the ratio at time $0 . \mathbf{d} \mathrm{mCa}^{2+}$ efflux rate, $n=3$ replicates per group; ${ }^{* *} p<0.01$, \#p $<0.05$. e FQ-PCR detected the mRNA expression levels of Mcu, Micul, and NClx in the heart after CLP for 12 h. $\mathbf{f}$ Western blotting was used to detect MCU, MICU1, and NCLX expression in hearts $12 \mathrm{~h}$ after CLP. GAPDH was used as a loading control

found that PINK1 expression increased in septic mouse cardiomyocytes after huMSC-exo treatment (Fig. 4a, FigS1. C), which further confirmed the relationship between PINK1 expression and NCLX activity. To further examine the source of increased PINK1 in cardiomyocytes after huMSC-exo treatment, we detected Pink1 mRNA in huMSCs and their exosomes and found that huMSC-exo carried more Pink1 mRNA than huMSCs (Fig. 4b, huMSC-exo group $4.225 \pm 0.1875$ versus huMSCs group $1(100 \%), p=0.0011)$.

We used Pink1-specific siRNA to inhibit Pink1 expression in huMSCs to confirm whether huMSC-exo increased PINK1 expression in recipient cardiomyocytes by transferring Pink1 mRNA from huMSCs. Pink1 siRNA dramatically inhibited Pink1 expression in huMSCs and their exosomes (Fig. 4c, huMSCs: Pink1siRNA group $0.1187 \pm 0.0421$ versus Neg siRNA $1.064 \pm$ $0.229, \quad p<0.0001$; huMSC-exo: Pink1-siRNA group $0.117 \pm 0.019$ versus Neg siRNA $1.249 \pm 0.281, p<$ 0.0001), and PINK1 expression in ACMs isolated from mice treated with Pink1-inhibited huMSC exosomes remained at a low level (Fig. 4d). These results suggest that the increase in PINK1 expression in recipient cardiomyocytes was due to the Pink1 mRNA carried in huMSC-exo.

HuMSC-exo with inhibited Pink1 could not reverse sepsisinduced ${ }_{\mathrm{m}} \mathrm{Ca}^{2+}$ efflux obstruction or mitochondrial damage

To further validate the relationship of PINK1 to NCLXmediated ${ }_{\mathrm{m}} \mathrm{Ca}^{2+}$ efflux, we monitored the ${ }_{\mathrm{m}} \mathrm{Ca}^{2+}$ efflux rate of ACMs from mice treated with Pink1-inhibited huMSC-exos. No significant difference was detected between the CLP + exo $^{\text {pink1 siRNA }}$ and CLP groups, but these groups were decreased significantly compared to the sham group (Fig. 5a, b, d, e, CLP + exo ${ }^{\text {pink1 siRNA }}$ groups $2.829 \pm 1.378$ versus CLP group $2.098 \pm 1.195$, $p=0.8505$ and sham group $5.836 \pm 0.4564, p=0.043)$. In contrast, the $\mathrm{mCa}^{2+}$ efflux rate in the CLP treated with exo $^{\mathrm{Neg}}$ siRNA group was the same as the sham group (Fig. 5a, c, e, sham group $5.836 \pm 0.4564$ versus CLP+ exo $^{\mathrm{Neg}}$ siRNA group $\left.5.988 \pm 1.192, p=0.998\right)$. These results suggest that the loss of Pink1 mRNA attenuated the ability of huMSC-exos to recover $\mathrm{mCa}^{2+}$ efflux. Whether this loss also weakened the ability of huMSCexos to protect mitochondria from sepsis-induced 


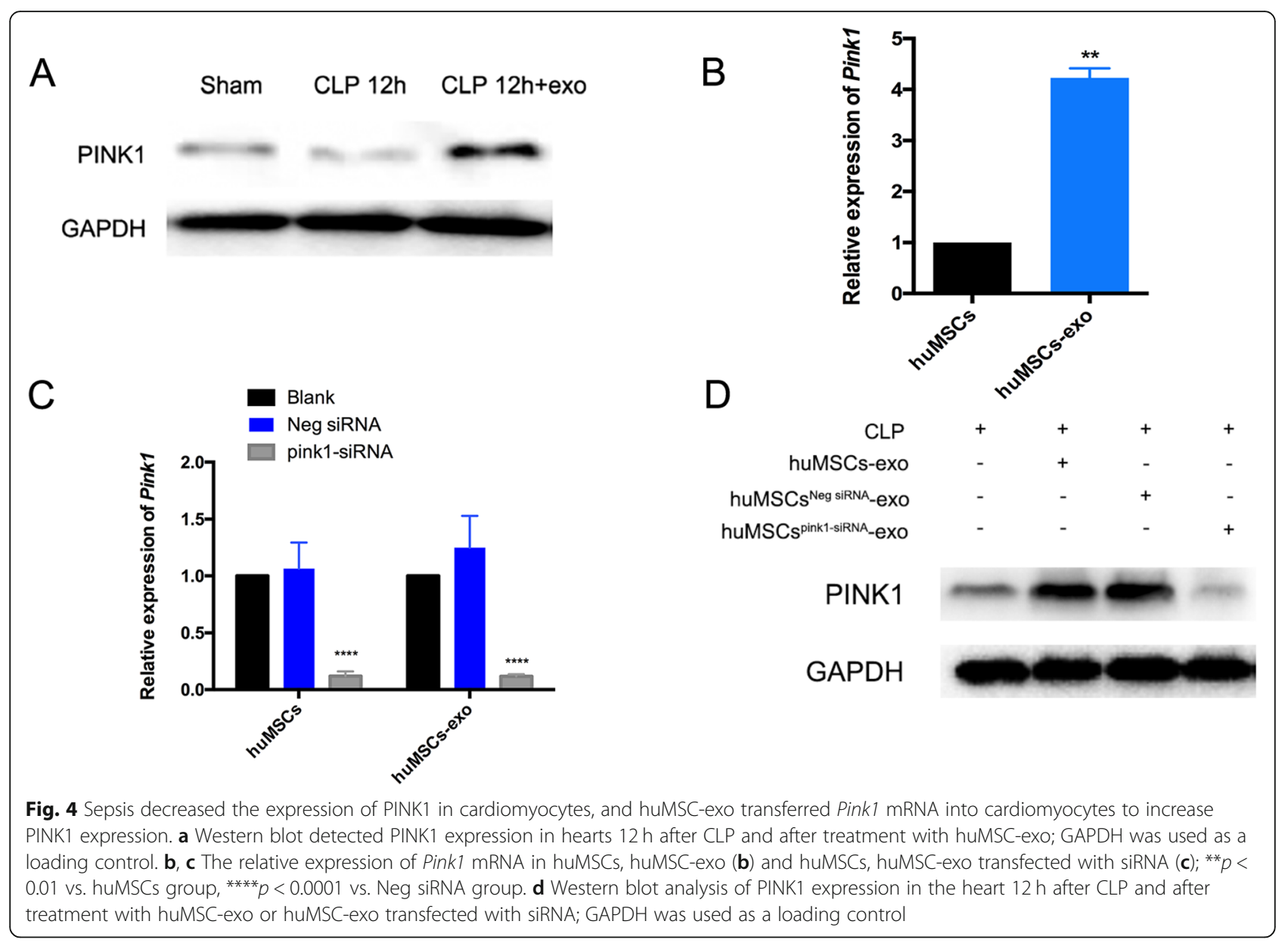

damage was not clear. We observed mitochondrial morphology using TEM and found that the mitochondrial cristae number still decreased and the arrangement was disordered in CLP mice with exo ${ }^{\text {pink1 siRNA }}$ treatment (Fig. 5f). This result indicates that the mitochondrial protection of huMSC-exos was greatly weakened after Pink1 mRNA inhibition.

\section{PINK1 may regulate NCLX-mediated ${ }_{\mathrm{m}} \mathrm{Ca}^{2+}$ efflux by affecting PKA activity after transfer from huMSC-exo to cardiomyocytes}

The above results confirmed the effect of PINK1 on NCLX-mediated $\mathrm{Ca}^{2+}$ efflux, but no evidence showed a direct interaction between PINK1 and NCLX. In contrast, many studies argued against a direct interaction of PINK1 and NCLX [13, 29]. One study showed that PINK1-deficient cells exhibited PKA inhibition and NCLX-mediated $\mathrm{mCa}^{2+}$ efflux impairment, which were fully rescued by activated PKA [13]. We also found that the PKA activity significantly reduced at CLP $12 \mathrm{~h}$ (FigS3. A). To determine whether PINK1 regulated NCLX-mediated ${ }_{m} \mathrm{Ca}^{2+}$ efflux via PKA, we used forskolin (FSK) and H89 to activate and inhibit PKA, respectively. The result showed that FSK could increase PKA activity significantly, and H89 could suppress PKA activity greatly (FigS3. B, C). AC16 cells were treated with LPS and huMSC-exo, and the ${ }_{\mathrm{m}} \mathrm{Ca}^{2+}$ efflux rate was monitored. The results showed that PKA activation by FSK (Fig. 6a, b light green) enhanced the ${ }_{\mathrm{m}} \mathrm{Ca}^{2+}$ efflux rate compared to treatment with huMSC-exos alone (Fig. 6a, b blue, LPS + exo + FSK group $6.15 \pm 1.03$ versus LPS + exo group $3.901 \pm 0.918, p=0.0448$ ), but the ${ }_{\mathrm{m}} \mathrm{Ca}^{2+}$ efflux rate decreased significantly when FSK and H89 were co-applied (Fig. 6a, b light orange, LPS + exo + FSK + H89 group $1.578 \pm 0.613$ versus LPS + exo + FSK group $6.15 \pm 1.03, p=0.0016$ ). Consistent with a previous study [13], we found that FSK greatly recovered the decrease in $\mathrm{mCa}^{2+}$ efflux rate caused by the absence of PINK1, but H89 abolished the effect of FSK (Fig. 6c, d, LPS + exo ${ }^{\text {pink1 }}$ siRNA + FSK group $4.283 \pm 1.145$ versus LPS + exo $^{\text {pink1 siRNA }}$ group 1.568 $\pm 0.676, p=0.0158$, and LPS + exo $^{\text {pink1 }}$ siRNA + FSK + H89 group $1.454 \pm 0.503$, $p=0.0131)$. The $\Delta \Psi \mathrm{m}$ also increased after FSK treatment, and this effect was eliminated by H89 (Fig. 6e, f, g, LPS + exo $^{\text {pink1 }}$ siRNA + FSK group $3.13 \pm 0.06$ versus LPS + exo $^{\text {pink1 siRNA }}$ group $2.882 \pm 0.108, p=0.0138$, and 


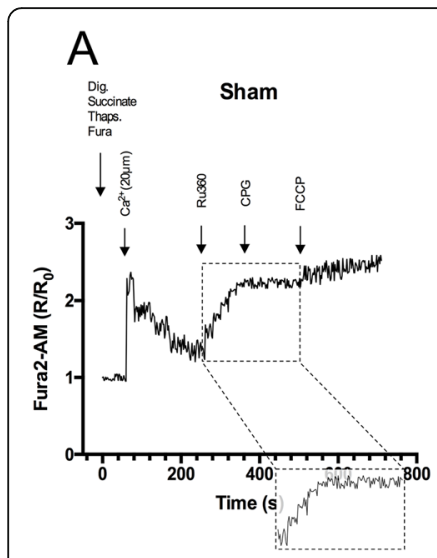

E

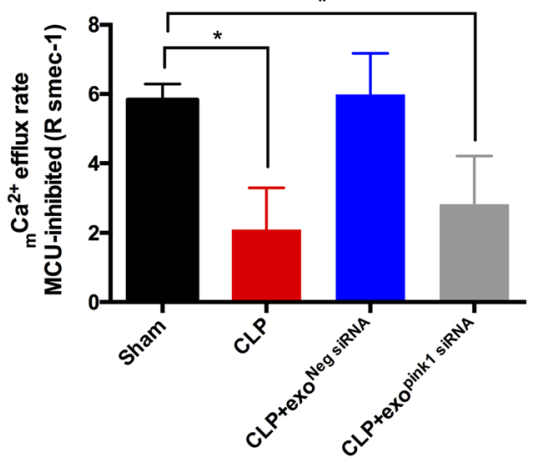

C

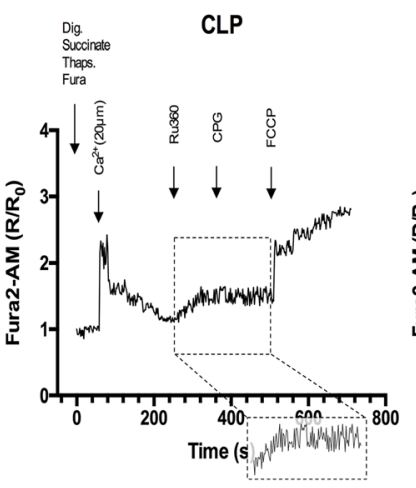

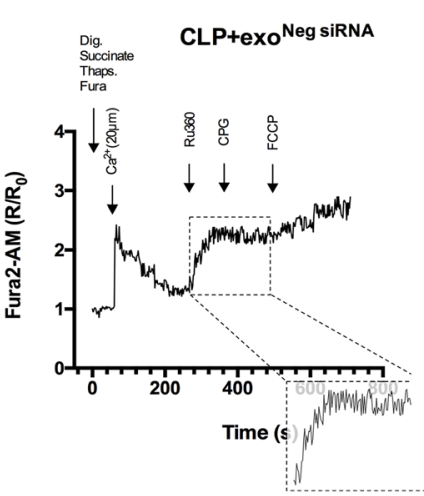

$\mathrm{D}$

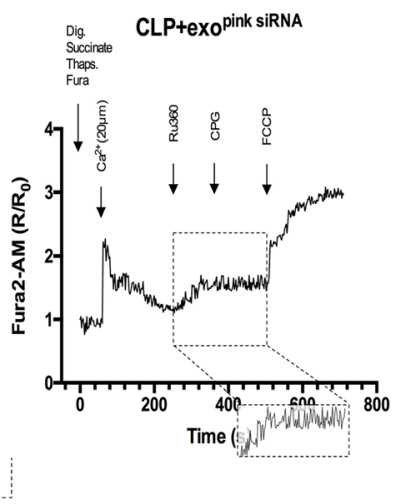

$\mathrm{F}$
CLP+exo $0^{\text {Neg siRNA }}$

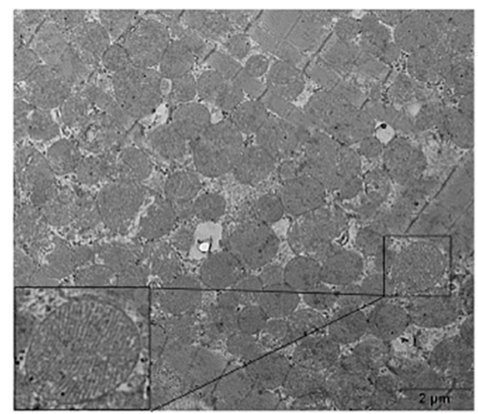

CLP+exo pink1 siRnA

Fig. 5 HuMSC-exo with inhibited PINK1 could not reverse sepsis-induced ${ }_{m} \mathrm{Ca}^{2+}$ efflux obstruction or mitochondrial damage. $\mathbf{a}, \mathbf{d}{ }_{\mathrm{m}} \mathrm{Ca}{ }^{2+}$ uptake and efflux in isolated permeabilized ACMs; dig.: digitonin, thaps.: thapsigargin, Ru360: MCU inhibitor, CGP: NCLX inhibitor; the box shows a magnified ${ }_{m} \mathrm{Ca}^{2+}$ efflux tracing (260 s-360 s); $\mathrm{R}$ indicates the ratio of the ratiometric reporter Fura-2 (340/380 nm excitation and $510 \mathrm{~nm}$ emission). $\mathrm{R} / \mathrm{R}_{0}$ indicates the ratio at each time point over the ratio at time $0 . \mathbf{e} \mathrm{m}^{\mathrm{Ca}}{ }^{2+}$ efflux rate, $n=3$ replicates per group, ${ }^{*} p<0.05$. $\mathbf{f}$ Mitochondrial architecture observed with TEM after treatment with siRNA-transfected huMSC-exo, imaged at a $\times 10,000$ magnification (scale bars, $2 \mu \mathrm{m}$ ), and the box in the image is a local magnification

LPS + exo ${ }^{\text {pink1 }}$ siRNA + FSK + H89 group $2.883 \pm 0.025$, $p=0.0141)$. These results suggest that PKA is essential for PINK1 regulation of NCLX-mediated ${ }_{\mathrm{m}} \mathrm{Ca}^{2+}$ efflux.

\section{Discussion}

The heart is an important target organ in severe sepsis/ septic shock, and its dysfunction manifests in many different ways in sepsis, including left and/or right ventricular impairment during systole or diastole, inadequate cardiac output and oxygen delivery, or primary myocardial cellular injury [30]. Sepsis with cardiac dysfunction increases mortality by $50 \%$ [31], which should arouse our attention. Fluid resuscitation is used clinically to improve cardiac perfusion, cardiac output, and systolic function at the early stage of sepsis to support the heart, but there is no specific treatment. This lack of strategy is likely due to the unclear and complicated mechanism of sepsis-induced cardiac dysfunction, which also explains the lack of drugs aimed at corresponding targets. Previous studies indicated that the main proposed mechanisms underlying the pathophysiology of myocardial dysfunction in sepsis support a prominent role of functional, rather than anatomical, abnormalities [31]. The clinically significant pathophysiological changes in cardiac function that occur early during sepsis may occur in the absence of cellular hypoxia or histological changes, which suggests that severe metabolic derangement underlies the development of septic cardiomyopathy [32-35]. This opinion is consistent with the results of the present study, which showed cardiomyocyte mitochondrial damage $12 \mathrm{~h}$ after CLP, which is much earlier than cardiomyocyte apoptosis $(24 \mathrm{~h}$ after CLP). This early damage explains our use of $12 \mathrm{~h}$ after CLP as the investigational time point. Mitochondrial damage is an important trigger that causes apoptosis, which is characterized by a change in mitochondrial architecture (swelling, internal vesicle formation, and abnormalities in cristae), mitochondrial DNA damage, and elevation in mitochondrial permeability transition $[9,36$, 37]. Mitochondria are the power houses of cells that provide continuous energy for heart activity, and injury inevitably induces metabolic derangement and a 


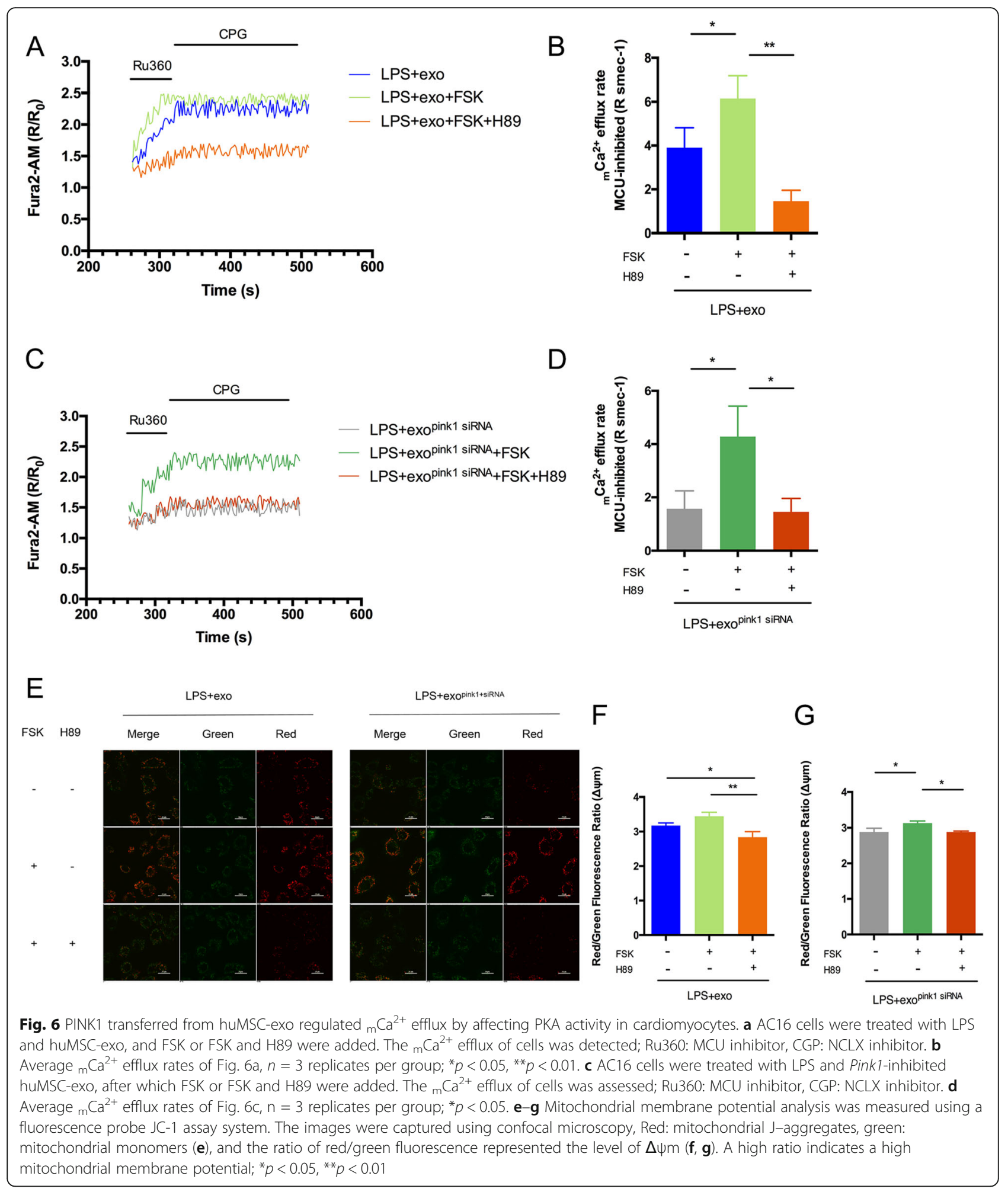

decrease in ATP production, which weakens myocardial contractility and eventually causes heart dysfunction.

The reported mechanisms of sepsis-induced cardiomyocyte mitochondrial dysfunction include energy metabolism disorder [38], calcium overload [39], autophagy
[40], and mitochondrial inner membrane damage [41]. Calcium acts as an important intracellular second messenger and plays a key role in cardiac contractility. Mitochondria are one of the largest calcium pools in cardiomyocytes and alleviate the high concentration of 
$\mathrm{Ca}^{2+}$ via the uptake of intracellular $\mathrm{Ca}^{2+}\left({ }_{\mathrm{i}} \mathrm{Ca}^{2+}\right)$ into the mitochondrial matrix. However, when $\mathrm{mCa}^{2+}$ exceeds full capacity, the mitochondrial permeability transition pore (MPTP) irreversibly open, and the $\Delta \Psi \mathrm{m}$ is reduced, which induces mitochondrial dysfunction [39]. Normal mitochondria need $\Delta \Psi \mathrm{m}$ to maintain their function, and a decreased $\Delta \Psi \mathrm{m}$ is an important cause of apoptosis. Calcium overload is an upstream event of mitochondrial depolarization [13]. Therefore, mitochondrial calcium overload may be an important mechanism of mitochondrial dysfunction in septic hearts. This hypothesis was further confirmed in the present study, which found cardiomyocyte impairment of $\mathrm{mCa}^{2+}$ shuttling $12 \mathrm{~h}$ after CLP, much earlier than apoptosis occurred.

There are two processes that cause mitochondrial calcium overload: excessive calcium uptake into mitochondria, which is mainly driven by MCU; or a decrease in calcium efflux from the mitochondrial matrix to the cytoplasm $[27,28]$. NCLX is the most important regulator of mitochondrial calcium efflux and may be the primary mechanism for $\mathrm{mCa}^{2+}$ extrusion in excitable cells [10]. Abnormal MCU or NCLX causes $\mathrm{mCa}^{2+}$ shuttling system disorder, but research showed that an $\mathrm{MCU}$ knockout yielded a relatively mild phenotype, and a conditional knockout of NCLX led to rapid fatal heart failure [28], which suggests that NCLX-mediated efflux is necessary to maintain homeostatic ${ }_{\mathrm{m}} \mathrm{Ca}^{2+}$ levels in cardiomyocytes and for survival. We found no change in $\mathrm{Ca}^{2+}$ uptake by cardiomyocyte mitochondria in the early stage of sepsis, but ${ }_{\mathrm{m}} \mathrm{Ca}^{2+}$ efflux was obviously abnormal. This result suggests that sepsis-induced cardiac dysfunction is caused by mitochondrial calcium overload resulting from reduced mitochondrial calcium efflux. However, no difference in NCLX expression was found between the CLP and sham group mice in our study, which suggests that the reason for dysfunction of $\mathrm{mCa}^{2+}$ efflux after CLP was related to the suppressed NCLX activity.

The present study found that expression of PINK1, a key protein associated with NCLX-mediated $\mathrm{mCa}^{2+}$ efflux, decreased significantly in CLP mouse hearts. PINK1 is a serine/threonine kinase that was initially linked to the pathogenesis of a familial form of Parkinson's disease $[42,43]$, and its loss-of-function mutations cause dopaminergic neuron mitochondrial calcium overload, which makes cells particularly vulnerable to injury [14]. PINK1 $\mathrm{KO}$ mice developed left ventricular dysfunction and cardiac hypertrophy, which led to pressure overloadinduced heart failure. Mitochondria from PINK1 KO hearts exhibited an altered morphology, reduced mitochondrial membrane potential, and decreased oxidative phosphorylation, which induced oxidative stress and increased cardiomyocyte apoptosis [42, 44, 45]. Although PINK1 is associated with NCLX-mediated ${ }_{m} \mathrm{Ca}^{2+}$ efflux, the interaction between PINK1 and NCLX is indirect [29]. Previous studies demonstrated that the loss of PINK1 led to PKA inhibition and modulation of the NCLX phosphorylation site by PKA activation was essential for NCLX activity [13, 46]. Our results also showed that sepsis decreased PKA activity. Therefore, we hypothesized that the PINK1-PKA-NCLX axis in cardiomyocytes regulates $\mathrm{mCa}^{2+}$ efflux, similar to neurons, and abnormalities in this axis cause sepsis-induced mitochondrial calcium overload in cardiomyocytes.

Next, we explored the role of huMSCs-exo in heart protection during sepsis and whether PINK1-PKANCLX axis may be a therapeutic target. Firstly, we confirmed that exosomes secreted from huMSCs attenuated sepsis-induced cardiomyocyte injury via mitochondrial repair. A more regular mitochondria morphology and greater ATP production means a more integrated structure and more powerful function. Secondly, our results showed that huMSC-exo reversed the sepsis-induced reduction in cardiomyocyte $\mathrm{mCa}^{2+}$ efflux. Notably, we found the huMSC-exo carried more Pink1 mRNA than the huMSCs themselves, and this mRNA was transferred from huMSC-exo to recipient cardiomyocytes to increase PINK1 expression. PKA activity was also increased after treatment with huMSC-exo. When Pink1 mRNA contained in huMSC-exo was inhibited, huMSCexo lost the ability of ${ }_{\mathrm{m}} \mathrm{Ca}^{2+}$ efflux regulation, and the PKA activity of recipient cardiomyocytes was not change in sepsis mouse hearts. Activated PKA rescued the LPSinduced reduction in $\mathrm{maa}^{2+}$ efflux and $\Delta \Psi \mathrm{m}$ when huMSC-exo lost their ability to regulate ${ }_{\mathrm{m}} \mathrm{Ca}^{2+}$ efflux, and this effect abolished after PKA activity inhibition by H89. These results suggest that huMSC-exos ameliorate sepsis-induced cardiomyocyte mitochondrial calcium overload via the transfer of Pink1 mRNA into cardiomyocytes to restore PINK1 expression, and upregulated PINK1 increases PKA activity to activate NCLXmediated $\mathrm{m}_{\mathrm{m}}{ }^{2+}$ efflux and protect cardiomyocytes from injury.

Research on PINK1 revealed that its multiple functions extend well beyond mitochondrial calcium efflux regulation. PINK1 is a crucial player in the mitochondrial quality control pathway [42, 47], and it promotes damaged mitochondria elimination via Parkin-dependent $[48,49]$ or Parkin-independent [42, 50] mitophagy. Therefore, in our research, after huMSCs-exo increase PINK1 expression in septic cardiomyocytes, is it possible that huMSCs-exo eliminate the damaged mitochondria caused by mitochondrial calcium overload via activation of PINK1-related mitophagy to protect cardiomyocytes? This hypothesis was not investigated in this study, but it provides direction for further research.

In conclusion, we first confirmed that sepsis-induced PINK1-PKA-NCLX axis abnormalities in cardiomyocytes 
are the main cause of cardiomyocyte mitochondrial calcium overload and heart dysfunction. We also found that exosomes secreted from huMSCs transferred Pink1 mRNA to cardiomyocytes and rescued the decrease in ${ }_{\mathrm{m}} \mathrm{Ca}^{2+}$ efflux-induced mitochondrial calcium overload by restoring the PINK1-PKA-NCLX axis. One important limitation of this study is that we did not examine whether PINK1-dependent mitophagy is involved in the cardioprotective effects of huMSC-exo. Nevertheless, our data support PINK1 as a therapeutic target to protect cardiomyocyte mitochondria, and the application of huMSC-exo is a promising strategy to combat sepsisinduced heart dysfunction.

\section{Abbreviations}

huMSCs: Human umbilical cord mesenchymal stem cells; CLP: Cecal ligation and puncture; TEM: Transmission electron microscope; huMSCsexo: Exosomes isolated from huMSCs; NCLX: Mitochondrial $\mathrm{Na}^{+} / \mathrm{Ca}^{2+}$ exchanger; PINK1: PTEN-induced putative kinase 1; HBDH: a-Hydroxybutyrate dehydrogenase; CK: Creatine kinase; CTnl: Cardiac troponin-l; FBS: Fetal bovine serum; PKA: Protein kinase A; EF: Ejection fraction; ACMs: Adult cardiomyocytes; SLP-2: Stomatin-like protein 2; PKC: Protein kinase C

\section{Supplementary Information}

The online version contains supplementary material available at https://doi. org/10.1186/s13287-021-02325-6.

\section{Additional file 1: Table S1. Index of Left Ventricular Function} measured by Echocardiography. Supplementary Figure 1. Quantitative analysis of protein expression. A: The exosome markers, ${ }^{*} p<0.05,{ }^{* * *} p<$ 0.001 compared with $3 \mu \mathrm{g}$ group; B: The expression of MCU, MICU1 and NCLX at $12 \mathrm{~h}$ after CLP and treatment with huMSCs-exo; C: The PINK1 expression at $12 \mathrm{~h}$ after CLP and treatment with huMSCs-exo, ${ }^{* *} p<0.01$ compared with sham group, \#\#\#\#p<0.0001 compared with CLP $12 \mathrm{~h}$ group; D: The PINK1 expression at $12 \mathrm{~h}$ after CLP and treatment with huMSCs-exo or Pink1 deficient huMSCs-exo, ${ }^{* * * *} p<0.0001$. Supplementary Figure 2. The PKH26-labeled exosomes were absorbed by cardiomyocytes (magnification: 400x). Red: PKH26, Green: cTnl, Blue: DAPI. Supplementary Figure 3. Detection of PKA activity. A: The PKA activity at $12 \mathrm{~h}$ after CLP and treatment with huMSCs-exo; B, C. The PKA activity after treated with FSK and $\mathrm{H} 89$.

\section{Acknowledgements}

We appreciate our colleagues for their valuable efforts on this paper.

\begin{abstract}
Authors' contributions
Hao Xu, Jing Zhu, and Jie Tian conceived of and designed the project. Qin Zhou, Min Xie, and Hao Xu perform the experiments and drafted the manuscript. Bin Tan contributed to the editing of the figures. Qin Yi performed the statistical analyses. Liang Ye and Yasha Li performed the sample collection. Yin Zhang and Xinyuan Zhang contributed to the cell culture, reagent procurement, and management. The authors read and approved the final manuscript.
\end{abstract}

\section{Funding}

This work was supported by the National Natural Science Foundation of China (Grant Number 81700250 and Grant Number 81670270). We thank the Department of Pediatric Research Institute for their technical assistance.

\section{Availability of data and materials}

The data that support the findings of this study are available from the corresponding author upon reasonable request.

\section{Declarations}

Ethics approval and consent to participate

The current study was approved by the Ethics Committee of the ChongQing Medical University.

\section{Consent for publication}

Not applicable.

\section{Competing interests}

The authors declare that there are no competing interests associated with the manuscript.

\section{Author details}

${ }^{1}$ Department of Pediatric Research Institute, Ministry of Education Key Laboratory of Child Development and Disorders, National Clinical Research Center for Child Health and Disorders (Chongqing), China International Science and Technology Cooperation base of Child development and Critical Disorders, Children's Hospital of Chongqing Medical University, Chongqing, People's Republic of China. ${ }^{2}$ Chongqing Key Laboratory of Pediatrics, Chongqing, People's Republic of China. ${ }^{3}$ Department of Cardiovascular (Internal Medicine), Ministry of Education Key Laboratory of Child Development and Disorders, National Clinical Research Center for Child Health and Disorders (Chongqing), China International Science and Technology Cooperation base of Child development and Critical Disorders, Children's Hospital of Chongqing Medical University, Chongqing, People's Republic of China. ${ }^{4}$ Department of Clinical Laboratory, Ministry of Education Key Laboratory of Child Development and Disorders, National Clinical Research Center for Child Health and Disorders (Chongqing), China International Science and Technology Cooperation base of Child development and Critical Disorders, Children's Hospital of Chongqing Medical University, Box 136, No. 3 Zhongshan RD, Yuzhong district, Chongqing 400014, People's Republic of China.

Received: 28 October 2020 Accepted: 3 April 2021

Published online: 06 May 2021

\section{References}

1. Zanotti-Cavazzoni SL, Hollenberg SM. Cardiac dysfunction in severe sepsis and septic shock. Curr Opin Crit Care. 2009;15(5):392-7. https://doi.org/10.1 097/MCC.0b013e3283307a4e.

2. Romero-Bermejo FJ, Ruiz-Bailen M, Gil-Cebrian J, Huertos-Ranchal MJ. Sepsis-induced cardiomyopathy. Curr Cardiol Rev. 2011;7(3):163-83. https:// doi.org/10.2174/157340311798220494.

3. Lichtenstern C, Brenner T, Bardenheuer HJ, Weigand MA. Predictors of survival in sepsis: what is the best inflammatory marker to measure? Curr Opin Infect Dis. 2012;25(3):328-36. https://doi.org/10.1097/QCO.0b013e3283 522038.

4. Iwata A, de Claro RA, Morgan-Stevenson VL, Tupper JC, Schwartz BR, Liu L, et al. Extracellular administration of BCL2 protein reduces apoptosis and improves survival in a murine model of sepsis. Plos One. 2011;6(2):e14729. https://doi.org/10.1371/journal.pone.0014729.

5. Mink SN, Kasian K, Jacobs H, Cheng ZQ, Light RB. N,N'-diacetylchitobiose, an inhibitor of lysozyme, reverses myocardial depression and lessens norepinephrine requirements in Escherichia coli sepsis in dogs. Shock. 2008; 29(6):681. https://doi.org/10.1097/SHK.0b013e31815816c3.

6. Lancel S, Hassoun SM, Favory R, Decoster B, Motterlini R, Neviere R. Carbon monoxide rescues mice from lethal sepsis by supporting mitochondrial energetic metabolism and activating mitochondrial biogenesis. J Pharmacol Exp Ther. 2009;329(2):641-8. https://doi.org/10.1124/jpet.108.148049.

7. Chopra M, Golden HB, Mullapudi S, Dowhan W, Dostal DE, Sharma AC. Modulation of myocardial mitochondrial mechanisms during severe polymicrobial sepsis in the rat. Plos One. 2011;6(6):e21285. https://doi.org/1 0.1371/journal.pone.0021285

8. Coalson JJ, Hinshaw LB, Guenter CA, Berrell EL, Greenfield LJ. Pathophysiologic responses of the subhuman primate in experimental septic shock. Lab Investig. 1975;32(4):561-9.

9. Martin L, Derwall M, Al Zoubi S, et al. The septic heart: current understanding of molecular mechanisms and clinical implications. Chest. 2019;155(2):427-37. https://doi.org/10.1016/j.chest.2018.08.1037. 
10. Luongo TS, Lambert JP, Gross P, Nwokedi M, Lombardi AA, Shanmughapriya $\mathrm{S}$, et al. The mitochondrial $\mathrm{Na}(+) / \mathrm{Ca}(2+)$ exchanger is essential for $\mathrm{Ca}(2+)$ homeostasis and viability. Nature. 2017;545(7652):93-7. https://doi.org/10.1038/nature22082.

11. Da Cruz S, De Marchi U, Frieden M, Parone PA, Martinou JC, Demaurex N. SLP-2 negatively modulates mitochondrial sodium-calcium exchange. Cell Calcium. 2010;47(1):11-8. https://doi.org/10.1016/j.ceca.2009.10.005.

12. Yang F, He XP, Russell J, Lu B. Ca2+ influx-independent synaptic potentiation mediated by mitochondrial $\mathrm{Na}(+)-\mathrm{Ca} 2+$ exchanger and protein kinase C. J Cell Biol. 2003;163(3):511-23. https://doi.org/10.1083/jcb.200307027.

13. Kostic M, Ludtmann MH, Bading $\mathrm{H}$, et al. PKA phosphorylation of NCLX reverses mitochondrial calcium overload and depolarization, promoting survival of PINK1-deficient dopaminergic neurons. Cell Rep. 2015;13(2):37686. https://doi.org/10.1016/j.celrep.2015.08.079.

14. Gandhi S, Wood-Kaczmar A, Yao Z, Plun-Favreau H, Deas E, Klupsch K, et al. PINK1-associated Parkinson's disease is caused by neuronal vulnerability to calcium-induced cell death. Mol Cell. 2009;33(5):627-38. https://doi.org/10.1 016/j.molcel.2009.02.013.

15. Kusadasi N, Groeneveld AB. A perspective on mesenchymal stromal cell transplantation in the treatment of sepsis. Shock. 2013;40(5):352-7. https:// doi.org/10.1097/SHK.0000000000000039.

16. Nemeth K, Leelahavanichkul A, Yuen PS, et al. Bone marrow stromal cells attenuate sepsis via prostaglandin E(2)-dependent reprogramming of host macrophages to increase their interleukin-10 production. Nat Med. 2009; 15(1):42-9. https://doi.org/10.1038/nm.1905.

17. Anderson P, Souza-Moreira L, Morell M, Caro M, O'Valle F, Gonzalez-Rey E, et al. Adipose-derived mesenchymal stromal cells induce immunomodulatory macrophages which protect from experimental colitis and sepsis. Gut. 2013;62(8):1131-41. https://doi.org/10.1136/gutjnl-2012-302152.

18. Krasnodembskaya A, Samarani G, Song Y, Zhuo H, Su X, Lee JW, et al. Human mesenchymal stem cells reduce mortality and bacteremia in gramnegative sepsis in mice in part by enhancing the phagocytic activity of blood monocytes. Am J Physiol Lung Cell Mol Physiol. 2012;302(10):L100313. https://doi.org/10.1152/ajplung.00180.2011

19. Weil BR, Herrmann JL, Abarbanell AM, Manukyan MC, Poynter JA, Meldrum DR. Intravenous infusion of mesenchymal stem cells is associated with improved myocardial function during endotoxemia. Shock. 2011;36(3):23541. https://doi.org/10.1097/SHK.0b013e318225f6ae.

20. Wang X, Gu H, Qin D, Yang L, Huang W, Essandoh K, et al. Exosomal miR-223 contributes to mesenchymal stem cell-elicited cardioprotection in polymicrobial sepsis. Sci Rep. 2015;5(1):13721. https://doi.org/10.1038/srep13721.

21. Ailawadi S, Wang X, Gu H, Fan GC. Pathologic function and therapeutic potential of exosomes in cardiovascular disease. Biochim Biophys Acta. 2015;1852(1):1-11. https://doi.org/10.1016/j.bbadis.2014.10.008.

22. Li T, Yan Y, Wang B, Qian H, Zhang X, Shen L, et al. Exosomes derived from human umbilical cord mesenchymal stem cells alleviate liver fibrosis. Stem Cells Dev. 2013;22(6):845-54. https://doi.org/10.1089/scd.2012.0395.

23. Xin H, Li Y, Liu Z, Wang X, Shang X, Cui Y, et al. MiR-133b promotes neural plasticity and functional recovery after treatment of stroke with multipotent mesenchymal stromal cells in rats via transfer of exosome-enriched extracellular particles. Stem Cells. 2013;31(12):2737-46. https://doi.org/10.1 002/stem.1409.

24. Rittirsch D, Huber-Lang MS, Flierl MA, Ward PA. Immunodesign of experimental sepsis by cecal ligation and puncture. Nat Protoc. 2009;4(1): 31-6. https://doi.org/10.1038/nprot.2008.214.

25. Ackers-Johnson M, Foo RS. Langendorff-free isolation and propagation of adult mouse cardiomyocytes. Methods Mol Biol. 1940;2019:193-204.

26. Thery C, Amigorena S, Raposo G, Clayton A. Isolation and characterization of exosomes from cell culture supernatants and biological fluids. Curr Protoc Cell Biol. 2006; Chapter 3:Unit 322.

27. Mallilankaraman K, Doonan P, Cardenas C, et al. MICU1 is an essential gatekeeper for MCU-mediated mitochondrial $\mathrm{Ca}(2+)$ uptake that regulates cell survival. Cell. 2012;151(3):630-44. https://doi.org/10.1016/j.cell.2012.10.011.

28. Kostic M, Sekler I. Functional properties and mode of regulation of the mitochondrial $\mathrm{Na}(+) / \mathrm{Ca}(2+)$ exchanger, NCLX. Semin Cell Dev Biol. 2019;94: 59-65. https://doi.org/10.1016/j.semcdb.2019.01.009.

29. Rakovic A, Grunewald A, Voges L, et al. PINK1-interacting proteins: proteomic analysis of overexpressed PINK1. Parkinsons Dis. 2011;2011:153979.

30. Beesley SJ, Weber G, Sarge T, Nikravan S, Grissom CK, Lanspa MJ, et al. Septic cardiomyopathy. Crit Care Med. 2018;46(4):625-34. https://doi.org/1 0.1097/CCM.0000000000002851.
31. Celes MR, Prado CM, Rossi MA. Sepsis: going to the heart of the matter. Pathobiology. 2013;80(2):70-86. https://doi.org/10.1159/000341640.

32. dos Santos CC, Gattas DJ, Tsoporis JN, Smeding L, Kabir G, Masoom H, et al. Sepsis-induced myocardial depression is associated with transcriptional changes in energy metabolism and contractile related genes: a physiological and gene expression-based approach. Crit Care Med. 2010; 38(3):894-902. https://doi.org/10.1097/CCM.0b013e3181ce4e50.

33. Finkel MS, Oddis CV, Jacob TD, Watkins SC, Hattler BG, Simmons RL. Negative inotropic effects of cytokines on the heart mediated by nitric oxide. Science. 1992;257(5068):387-9. https://doi.org/10.1126/science.1631560.

34. Hotchkiss RS, Karl IE. Reevaluation of the role of cellular hypoxia and bioenergetic failure in sepsis. JAMA. 1992;267(11):1503-10. https://doi.org/1 0.1001/jama.1992.03480110079038.

35. Piper RD, Li FY, Myers ML, Sibbald WJ. Structure-function relationships in the septic rat heart. Am J Respir Crit Care Med. 1997;156(5):1473-82. https://doi. org/10.1164/ajrccm.156.5.96-10085.

36. Suliman HB, Carraway MS, Piantadosi CA. Postlipopolysaccharide oxidative damage of mitochondrial DNA. Am J Respir Crit Care Med. 2003;167(4):570 9. https://doi.org/10.1164/rccm.200206-5180C.

37. Brealey D, Brand M, Hargreaves I, Heales S, Land J, Smolenski R, et al. Association between mitochondrial dysfunction and severity and outcome of septic shock. Lancet. 2002;360(9328):219-23. https://doi.org/10.1016/S014 0-6736(02)09459-X.

38. Drosatos K, Pollak NM, Pol CJ, Ntziachristos P, Willecke F, Valenti MC, et al. Cardiac myocyte KLF5 regulates Ppara expression and cardiac function. Circ Res. 2016:118(2):241-53. https://doi.org/10.1161/CIRCRESAHA.115.306383.

39. Yarana C, Sripetchwandee J, Sanit J, Chattipakorn S, Chattipakorn N. Calcium-induced cardiac mitochondrial dysfunction is predominantly mediated by cyclosporine A-dependent mitochondrial permeability transition pore. Arch Med Res. 2012;43(5):333-8. https://doi.org/10.1016/j.a rcmed.2012.06.010.

40. Piquereau J, Godin R, Deschenes S, et al. Protective role of PARK2/Parkin in sepsis-induced cardiac contractile and mitochondrial dysfunction. Autophagy. 2013;9(11):1837-51. https://doi.org/10.4161/auto.26502.

41. Smeding L, Plotz FB, Groeneveld AB, Kneyber MC. Structural changes of the heart during severe sepsis or septic shock. Shock. 2012;37(5):449-56. https:// doi.org/10.1097/SHK.0b013e31824c3238.

42. Arena $G$, Valente EM. PINK1 in the limelight: multiple functions of an eclectic protein in human health and disease. J Pathol. 2017;241(2):251-63. https://doi.org/10.1002/path.4815.

43. Valente EM, Abou-Sleiman PM, Caputo V, Muqit MM, Harvey K, Gispert S, et al. Hereditary early-onset Parkinson's disease caused by mutations in PINK1. Science. 2004;304(5674):1158-60. https://doi.org/10.1126/science.1 096284

44. Billia F, Hauck L, Konecny F, Rao V, Shen J, Mak TW. PTEN-inducible kinase 1 (PINK1)/Park6 is indispensable for normal heart function. Proc Natl Acad Sci U S A. 2011;108(23):9572-7. https://doi.org/10.1073/pnas.1106291108.

45. Siddall HK, Yellon DM, Ong SB, Mukherjee UA, Burke N, Hall AR, et al. Loss of PINK1 increases the heart's vulnerability to ischemia-reperfusion injury. PLoS One. 2013;8(4):e62400. https://doi.org/10.1371/journal.pone.0062400.

46. Dagda RK, Pien I, Wang R, Zhu J, Wang KZQ, Callio J, et al. Beyond the mitochondrion: cytosolic PINK1 remodels dendrites through protein kinase A. J Neurochem. 2014;128(6):864-77. https://doi.org/10.1111/jnc.12494.

47. Okatsu K, Oka T, Iguchi M, Imamura K, Kosako H, Tani N, et al. PINK1 autophosphorylation upon membrane potential dissipation is essential for Parkin recruitment to damaged mitochondria. Nat Commun. 2012;3(1):1016. https://doi.org/10.1038/ncomms2016.

48. Rakovic A, Grunewald A, Kottwitz J, et al. Mutations in PINK1 and Parkin impair ubiquitination of Mitofusins in human fibroblasts. Plos One. 2011;6(3): e16746. https://doi.org/10.1371/journal.pone.0016746.

49. Seibler $\mathrm{P}$, Graziotto J, Jeong H, Simunovic F, Klein C, Krainc D. Mitochondrial Parkin recruitment is impaired in neurons derived from mutant PINK1 induced pluripotent stem cells. J Neurosci. 2011;31(16):5970-6. https://doi. org/10.1523/JNEUROSCI.4441-10.2011.

50. Lazarou M, Sliter DA, Kane LA, Sarraf SA, Wang C, Burman JL, et al. The ubiquitin kinase PINK1 recruits autophagy receptors to induce mitophagy. Nature. 2015;524(7565):309-14. https://doi.org/10.1038/nature14893.

\section{Publisher's Note}

Springer Nature remains neutral with regard to jurisdictional claims in published maps and institutional affiliations. 